\title{
Research Square
In vitro validation of the immunogenicity of the predicted neoepitopes from high-risk estrogen receptor-positive breast cancer
}

\author{
Yun-jeong Choe \\ Yonsei University College of Medicine \\ Eunyoung Kim \\ Yonsei University College of Medicine \\ Jooyeon Oh \\ Yonsei University College of Medicine \\ Miran Jang \\ Yonsei University College of Medicine \\ Weixan Fu \\ University of Pennsylvania \\ Hann Lee \\ Yonsei University College of Medicine \\ Minho Chung \\ Theragen Bio \\ Kyung-Ho Pyo \\ Yonsei University College of Medicine \\ Chung-Bong Synn \\ Yonsei University College of Medicine \\ Sora Kim \\ Yonsei University College of Medicine \\ Yohan Yang \\ Yonsei University College of Medicine \\ Ahyeon Kim \\ Yonsei University College of Medicine \\ Byung Chul Cho \\ Yonsei University College of Medicine \\ Han Sang Kim \\ Yonsei University College of Medicine \\ Sangwoo Kim \\ Yonsei University College of Medicine \\ Beatriz Carreno \\ University of Pennsylvania \\ Jee Ye Kim \\ Yonsei University College of Medicine \\ Soonmyung Paik ( $\nabla$ soonmyungpaik@yuhs.ac) \\ Yonsei University College of Medicine
}

\section{Research Article}

Keywords: breast cancer, neoepitope vaccination, estrogen receptor

Posted Date: November 15th, 2021

DOI: https://doi.org/10.21203/rs.3.rs-1041598/v1

License: @ (i) This work is licensed under a Creative Commons Attribution 4.0 International License. Read Full License 


\section{Abstract}

Whether estrogen receptor-positive (ER+) breast cancer $(\mathrm{BC})$ can be a target for therapeutic neoepitope vaccination is not clear due to its low mutation burden.

We tested the immunogenicity of predicted neoepitopes from exome and RNA-seq data from three ER+/luminal B subtype BC samples using IFN-y ELISpot assays of HLA-matched donor PBMCs. As a control, three ER- BC and three lung cancers were tested. The ensemble of Neopepsee and pVACseq pipelines predicted 93 neoepitopes from 299 SNVs in three ER+ BCs. Among them, 90 could be tested with ELISpot, and 14 (15.6\%) were immunogenic (1, 5, and 10 for each tumor). In three ER- BC samples, 52 neoepitopes were predicted from 271 SNVs, and 12 (25.0\%) of 48 tested were immunogenic (2, 4, and 8 for each tumor). Of the three lung cancers, 53 of 72 predicted neoepitope candidates were tested, and 10 of them were immunogenic (18.9\%) $(0,1$, and 11 for each tumor). These differences were not statistically significant.

We conclude that luminal B subtype BCs express neoepitopes and can be a candidate for therapeutic neoepitope vaccination.

\section{Introduction}

Cancer results from the accumulation of somatic mutations. ${ }^{1}$ The Cancer Genome Atlas (TCGA) results demonstrated that most solid tumor cells have an average of 50 nonsynonymous mutations. ${ }^{2}$ Minor fractions of peptides encoded by somatic mutations in cancer cells are presented by major histocompatibility complex (MHC) class I proteins and induce an antitumor immune response by T lymphocytes. ${ }^{3}$ Such mutant peptides are called "neoepitopes".

Neoepitopes are thought to be the best targets for therapeutic cancer vaccines since, unlike nonmutated tumor-associated antigens, they are expected to induce a robust T-cell immune response due to a lack of central tolerance and are unlikely to induce autoimmunity to normal cells. ${ }^{4-6}$ Adoptive transfer of $e x$ vivo expanded tumor-infiltrating lymphocytes enriched for neoepitope-specific T cells resulted in remarkable responses in some patients, especially when administered together with immune checkpoint inhibitors, demonstrating that mutated neoepitopes are valid therapeutic targets. ${ }^{7}$

Early results from dendritic cell, mRNA, or peptide vaccination against neoepitopes demonstrated induction of antitumor immunity and evidence of epitope

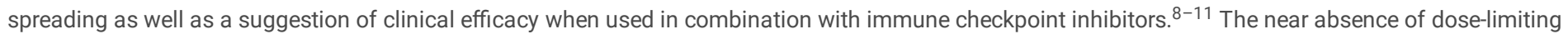
toxicities due to off-target immune responses against normal tissue in both therapeutic vaccine and adoptive cell transfer trials is reassuring. ${ }^{8-11}$ With the advancement of timely identification of neopeptide candidates, many clinical trials are now ongoing with a variety of vaccine platforms targeting neoepitopes. ${ }^{4,5,12}$

While multiple neoepitope vaccine trials are ongoing for solid tumors, ER+ BC patients are usually not targeted due to their lower mutation burden and hence lower predicted neoepitope burden. ${ }^{13-15}$ Among ER+ BC, the luminal B subtype is characterized by a high proliferation rate and poor outcome. ${ }^{16}$ Although they are sensitive to chemotherapy, their clinical outcome is still worse than that of other ER+ BC (luminal A subtype) even after chemotherapy. ${ }^{17,18}$ In the TAILORx trial for node-negative ER+ BC patients, $15 \%$ of patients with OncotypeDx recurrence scores over 25 (i.e., luminal B) experienced recurrence at a distant site within 9 years even after chemoendocrine therapy. ${ }^{19}$ Prognosis after distant recurrence events is dire with no curative therapy available. ${ }^{20}$ Therefore, it is worthwhile to explore neoepitope-directed immunotherapy in this disease population. Although many studies have reported the neoepitope landscape of BC, most of them lack experimental validation of the immunogenicity of the predicted neoepitopes. ${ }^{21-23}$ When considering the low positive predictive value of the prediction algorithms employed in those studies, ${ }^{24}$ it is reasonable to conclude that the true immunogenicity of ER+ BC is largely unknown. In this study, we tested the immunogenicity of predicted neoepitopes from 3 ER+ luminal B BCs using an ELISpot assay with HLA-matched PBMCs from three healthy donors. ${ }^{25,26}$ As a comparator, we included 3 ER- BC and 3 PDXs derived from lung cancers.

Previously, we reported a neoepitope prediction model, "Neopepsee", which was trained with multiple features that may influence the immunogenicity of mutated peptides. ${ }^{27}$ Although Neopepsee showed good performance compared to previously reported algorithms in a validation set in silico, experimental validation was lacking. Therefore, as an integral secondary aim, we compared the positive predictive value of Neopepsee in comparison to that of pVACseq. ${ }^{28}$

Another secondary aim was to examine whether whole-exome and RNAseq-based HLA typing are reliable by comparing the results with two-digit clinical HLA genotyping and dedicated NGS-based HLA typing kits.

\section{Methods}

\section{Subjects and ethics requirements}

Fresh tumor and peripheral blood samples were obtained from six breast cancer patients undergoing surgery at Yonsei Cancer Center with written informed consent and institutional review board approval (Severance Hospital IRB approval number: 4-2017-0715). All patients except one had not received any systemic treatment or radiotherapy in the biopsied area before surgery.

Anonymized patient-derived xenografts (archived frozen at F4 generation) with paired peripheral blood samples were available for three lung cancer patients with informed consent (Severance Hospital IRB approval number 4-2016-0788). Leukoreduction system (LRS) chamber blood cells from 50 healthy donors were obtained from the central blood bank of Korea (Severance Hospital IRB approval number: 4-2018-0803).

All methods were performed in accordance with the relevant guidelines and regulations.

Page $2 / 10$ 


\section{HLA genotyping}

We used Sanger sequencing-based clinical HLA genotyping results with two-digit resolution (BIOWITHUS Inc. Seoul, Korea). In addition, we applied Optitype 29 and HLAminer ${ }^{30}$ and used the Omixon Holotype HLA ${ }^{T M}$ kit (Omixon Biocomputing Ltd, Budapest, Hungary) to evaluate NGS-based HLA typing methods.

\section{Neoepitope candidate selection}

Paired whole-exome sequencing (WES) and RNA sequencing were performed at Yonsei Genome Center. A WES library was generated using SureSelect Exome V7 (Agilent Technologies, CA, USA) for breast cancer samples or V5 for lung cancer samples, and sequencing data were produced using Novaseq6000 (Illumina, CA, USA). Multiplexing for sequencing was designed to acquire more than 200X depth for tumor samples and 100X depth for matched normal samples. A total RNA sequencing library was generated using a TruSeq Stranded Total RNA Library Prep Kit (Illumina), and sequencing data were produced using NovaSeq 6000 (except for sample Neo 1, which was sequenced using NextSeq 550) to acquire more than 5 G data for each sample.

Tumor-normal paired WES reads were aligned to the human reference genome with BWA-MEM. ${ }^{31}$ To reduce mouse contamination from patient-derived xenograft (PDX) sample sequencing data, we made a concatenated reference of mice and humans as recommended and performed an alignment with concatRef on the PDX of 3 lung cancer patients. ${ }^{32}$ We used Picard (version 2.19) (https://broadinstitute.github.io/picard/) to sort the sequencing reads in order of genomic coordinates, to remove PCR duplicates and to fix the mate information of the paired-end sequence read.

Somatic mutations were identified via local assembly of haplotypes using Mutect2. ${ }^{33}$ Following the GATK Best Practices recommendation, FilterMutectCalls GATK4 (version 4.0.8.0.1) was used for confident somatic calls, reads with base quality lower than 30 were removed, and germline variants were filtered with gnomAD. ${ }^{34}$ We selected missense mutations with more than 20 depth and more than five alternative allele counts and manually reviewed calls with Integrative Genomics Viewer (IGV).

The patient-derived reliable somatic mutations and patient HLA genotyping information and RNA sequencing data were prepared as input for NoePepsee to predict patient-specific neoepitope candidates. ${ }^{27}$ NeoPepsee classifies potential neoantigens into three categories concerning predicted immunogenicity: high, medium, and low levels. Peptides at the high and medium levels were selected as candidates. The pVACseq pipeline was run at the Carreno laboratory at the University of Pennsylvania as reported with modified selection criteria (IC50<100 nM instead of $500 \mathrm{nM}, \mathrm{DNA}$ VAF>20, and RNA VAF>0). ${ }^{28}$

\section{Processing of donor blood}

Leukoreduction system (LRS) chambers from anonymous donors were obtained from the central bank. After removing 500 microliters for clinical HLA typing, a buffy coat was collected after Ficoll gradient centrifugation at 2,000 rpm for 25 minutes at room temperature. The buffy coat was washed in PBS and resuspended in CellBanker2 solution (AMS Biotechnology Ltd, Abingdon, U.K.) and stored in the deep freezer.

\section{Clinical HLA genotyping of donor blood}

DNA was extracted from $300 \mu \mathrm{L}$ of whole blood using the Gentra Puregene Blood Kit (QIAGEN, Hilden, Germany). Alternatively, DNA from PBMCs was isolated using the DNeasy Blood \& Tissue Kit (QIAGEN). DNA was eluted in $30 \mu \mathrm{L}$ nuclease-free water, and samples were stored in a $-80^{\circ} \mathrm{C}$ deep freezer until analysis. Sequences were analyzed with BIOWITHUS Inc. (Seoul, Korea).

\section{Peptide synthesis}

All peptides were synthesized, HPLC purified to over $98 \%$ by AnyGen (Gwangju, Korea) and resolved in DMSO at a stock concentration of 10 mg/ml. A working concentration of $10 \mu \mathrm{g} / \mathrm{ml}$ was used for the ELISpot assay.

\section{ELISpot assay}

White blood cells from HLA-matched donors were subjected to an ELISpot assay. An IFN-囚 precoated ELISpot assay kit (Cellular Technology Limited,

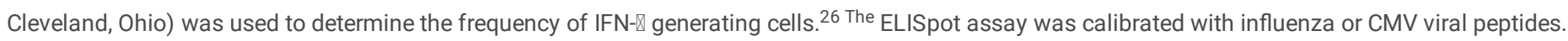

Briefly, frozen PBMCs isolated from LRS chambers were thawed and rested overnight in culture medium consisting of CTS optimizer T cell expansion medium SFM (Gibco), human serum (5\%), and 20 Unit Interleukin-2 (Gibco). PBMCs were plated in U-bottom 96-well plates (105 cells per well) in culture medium. PBMCs in each well were incubated with the corresponding peptide $(10 \mu \mathrm{g} / \mathrm{ml})$ at three-day intervals for peptide stimulation. After three cycles of peptide stimulation, stimulated PBMCs were replated onto IFN- - -coated ELISpot plates ( $2 \times 10^{5}$ cells per well) followed by peptide restimulation for 24 hours. After restimulation with the peptide (on day 10), IFN- $\$ production by the specific T-cell response to each peptide was estimated by ELISpot assay according to the manufacturer's instructions. Peptide-stimulated PBMCs were evaluated and compared with the media (no-peptide) control, and phytohemagglutinin (PHA) was used as a positive control. An ELISpot CTL reader was used for scanning the ELISpot assay plate, and Immunospot software was used to analyze spot information.

Since there were multiple alleles expressed in each donor blood, we used three donors for each test, and a peptide was called a true neoepitope only when more than two of the 3 HLA-matched donor PBMCs showed spot counts greater than $20 \%$ above the no peptide control upon rechallenge with the peptide after ten days of incubation with the peptide and cytokines. PHA was used as a positive control.

\section{Result}


Among six primary breast cancer patients, 3 were clinically triple-negative subtypes (Case ID. NEO 1,2,6; Table 1), a type of breast cancer with negative expression of estrogen, progesterone, and human epidermal growth factor receptor-2 (HER2), and 3 were hormone-receptor-positive breast cancer with luminal B subtype with high Ki67 labeling index (Case ID, NEO 3,4,5; Table 1). In hormone receptor-positive breast cancer patients, 2 showed invasive lobular carcinoma histology. At the time of sample collection, patients were not undergoing therapy except one patient (NEO2). NEO2 patients had received neoadjuvant chemotherapy (AC [doxorubicin/cyclophosphamide]) followed by a weekly paclitaxel regimen but withdrew neoadjuvant chemotherapy after one paclitaxel infusion due to early disease progression and underwent surgery.

Two PDXs established from surgical specimens from squamous cell carcinoma of the lung (AV10 and AV38) and one from non-small-cell lung cancer (AV10) were used to represent lung cancer (Table 1).

Table 1

Patient demographics.

\begin{tabular}{|c|c|c|c|c|c|c|c|c|}
\hline $\begin{array}{l}\text { Case } \\
\text { ID }\end{array}$ & Age & Histologic Type & $\begin{array}{l}\text { Histologic } \\
\text { Grade }\end{array}$ & $\begin{array}{l}\text { Ki67 } \\
(\%)\end{array}$ & $\begin{array}{l}\text { Sample } \\
\text { source }\end{array}$ & Subtype & Stage & HLA type \\
\hline NE01 & 60 & IDC & 3 & 77.2 & Surgery & TNBC & Stage IIA (pT1N1M0) & $\begin{array}{l}A^{\star} 24: 02, \\
A^{*} 33: 03\end{array}$ \\
\hline NEO2 & 47 & IDC & 3 & 66.1 & Surgery & TNBC & $\begin{array}{l}\text { ypStage IA } \\
\text { (ypT1N0M0) }\end{array}$ & $\begin{array}{l}A * 24: 02 \\
A * 33: 03\end{array}$ \\
\hline NEO3 & 74 & IDC & 2 & 63.4 & Surgery & $\begin{array}{l}\text { Luminal } B \\
B C\end{array}$ & Stage IIA (pT2NOM0) & $\begin{array}{l}A^{\star} 02: 06 \\
A^{\star} 11: 01\end{array}$ \\
\hline NEO4 & 52 & ILC & 2 & 55.6 & Surgery & $\begin{array}{l}\text { Luminal B } \\
\text { BC }\end{array}$ & Stage IIA (pT2NOM0) & $\begin{array}{l}A^{\star} 02: 06 \\
A^{\star} 11: 01\end{array}$ \\
\hline NEO5 & 52 & ILC & 2 & 47.2 & Surgery & $\begin{array}{l}\text { Luminal B } \\
\text { BC }\end{array}$ & Stage IIA (pT2NOM0) & $\begin{array}{l}A^{\star} 02: 01 \\
A^{\star} 02: 01\end{array}$ \\
\hline NEO6 & 74 & IDC & 3 & 82.3 & Surgery & TNBC & Stage IIA (pT2NOM0) & $\begin{array}{l}A^{\star} 02: 01 \\
A^{\star} 24: 02\end{array}$ \\
\hline AV10 & NA & $\begin{array}{l}\text { Squamous cell } \\
\text { carcinoma }\end{array}$ & NA & NA & PDX & SQCC & stage IIB (T2bN1M0) & $\begin{array}{l}A \star 32: 01 \\
A * 33: 03\end{array}$ \\
\hline AV13 & NA & Non-small cell & NA & NA & PDX & NSCLC & stage IIIA (T2bN2 M) & $\begin{array}{l}A^{\star} 02: 01 \\
A^{\star} 30: 04\end{array}$ \\
\hline AV38 & NA & $\begin{array}{l}\text { Squamous cell } \\
\text { carcinoma }\end{array}$ & NA & NA & PDX & SQCC & stage $1 b$ & $\begin{array}{l}A * 01: 01 \\
A * 31: 01\end{array}$ \\
\hline
\end{tabular}

\section{Comparison of HLA genotyping methods}

We used Sanger sequencing-based clinical HLA genotyping results with two-digit resolution (BIOWITHUS Inc. Seoul, Korea). In addition, we applied Optitype 29 and HLAminer ${ }^{30}$ and used the Omixon Holotype HLA ${ }^{T M}$ kit (Omixon Biocomputing Ltd, Budapest, Hungary) to evaluate NGS-based HLA typing methods (Table 2). 
Table 2

HLA genotyping results comparison among three different methods.

\begin{tabular}{|c|c|c|c|c|}
\hline Case ID & $\begin{array}{l}\text { HLA-A genotype } \\
\text { (clinical) }\end{array}$ & OptiType & HLAminer & Omixon Holotype \\
\hline NE01 & $A * 24: 02, A * 33: 03$ & $A * 24: 02, A * 33: 03$ & $A * 24: 02, A * 33: 03$ & $A^{*} 24: 02, A^{*} 33: 03$ \\
\hline NEO2 & $A^{*} 24: 02, A^{\star} 33: 03$ & $A * 24: 02, A * 33: 03$ & $A^{\star} 24: 02, A^{\star} 33: 03$ & $A * 24: 02, A * 33: 03$ \\
\hline NEO3 & $A * 02: 06, A * 11: 01$ & $A^{*} 02: 06, A^{*} 11: 01$ & $A^{\star} 02: 06, A^{\star} 11: 01$ & $A^{*} 02: 06, A^{*} 11: 01$ \\
\hline NEO4 & $A^{*} 02: 06, A^{*} 11: 01$ & $A^{*} 02: 06, A^{*} 11: 01$ & $A^{\star} 02: 06, A^{\star} 11: 01$ & $A^{*} 02: 06, A^{*} 11: 01$ \\
\hline NEO5 & $A^{*} 02: 01, A^{*} 02: 01$ & $A^{*} 02: 01, A^{*} 02: 01$ & $A^{\star} 02: 01, A^{\star} 02: 01$ & $A * 02: 01, A * 02: 01$ \\
\hline NEO6 & $A^{*} 02: 01, A^{*} 24: 02$ & $A^{*} 02: 01, A^{*} 24: 02$ & $A * 24: 95, A^{* 69: 01}$ & $A^{*} 02: 01, A^{*} 24: 02$ \\
\hline AV10 & $A^{\star} 32: 01, A^{\star} 33: 03$ & $A * 33: 03, A * 33: 03$ & $A * 33: 147, N A$ & ND \\
\hline AV13 & $A^{\star} 02: 01, A^{\star 30: 04}$ & $A^{\star} 02: 01, A^{\star} 30: 04$ & $A * 30: 04, A^{* 69: 01}$ & ND \\
\hline AV38 & $A * 01: 01, A * 31: 01$ & $A^{*} 01: 01, A^{*} 31: 01$ & $A^{* 01: 01, A * 36: 03}$ & ND \\
\hline Average accuracy & reference & $17 / 18(94 \%)$ & $11 / 18(61 \%)$ & $12 / 12(100 \%)$ \\
\hline \multicolumn{5}{|c|}{ Note: bold words represent mismatches } \\
\hline \multicolumn{5}{|c|}{$\begin{array}{l}\text { There was a high concordance between NGS-based Optitype and clinical HLA genotyping tests with } 94 \% \text { agreement (Supplementary Table } 1) \text {. However, the } \\
\text { concordance rate for HLAminer was low (61\%). Omixon Holotype assay results were } 100 \% \text { concordant with clinical genotypes. }\end{array}$} \\
\hline
\end{tabular}

\section{Candidate neoepitope selection}

Paired whole-exome sequencing of tumor and PBMC DNA yielded an average of 95 (range 70 to 107) nonsynonymous somatic mutations in breast cancer tumors and an average of 109 (range 76 to 143) nonsynonymous mutations in lung cancer PDX samples.

In this study, we focused only on HLA-A allele-restricted neoepitopes for experimental validation. For each tumor sample, all candidate epitopes predicted by Neopepsee and pVACseq are listed in Supplementary Table 1, with summarized ELISpot results presented in Table 3. Neopepsee considers only SNVs and does not consider fusion genes or intron retention. Neopepsee selected an average of 19 candidates (range 7 to 33 ) with breast cancer mutations and 17 candidates (range 11 to 21 ) with lung cancer mutations. For pVACseq analysis with a modified threshold of IC50 below $100 \mathrm{nM}$, DNA VAF >20 and RNA VAF $>0$ yielded an average of 9 best candidates for breast cancer (range 3 to 26) and 15 for lung cancer PDX samples (range 8 to 21).

In total, Neopepsee predicted 159 neoepitope candidates from 898 mutations (17.7\%), and pVACseq predicted 84 (9.4\%), with only 26 (2.9\%) shared between the two prediction algorithms. Thus, the ensemble of Neopepsee and pVACseq identified 217 candidate neoepitopes (24.2\%) from 898 SNVs.

In vitro validation of candidate neoepitopes

Based on a report by Stronen et al, ${ }^{25,26}$ we used HLA-matched donor blood for an ELISpot assay of IFN- $\square$ secretion from neoantigen-specific T cell populations to validate candidate neoepitopes. ${ }^{25,26}$ Among 217 candidate neoepitopes, 22 Neopepsee candidate peptides and 9 pVACseq candidate peptides could not be tested either due to synthesis or purification failure or due to lack of donor blood for the specific HLA alleles. In total, 36 of 191 tested candidates (18.8\%) were positive by ELISpot. The results summarized by case and prediction algorithms are provided in Table 3, with detailed information for the immunogenic peptides provided in Table 4. For some candidate peptides, although no memory response could be demonstrated, there were higher numbers of spots compared to the wells not stimulated with the peptide from day 0 . We did not consider those peptides to be positive, although some reports in the literature considered such results to be immunogenic. For some peptides, there were strong responses in only one of the three donor blood samples, suggesting that the response could be directed toward the allele other than the predicted allele.

For our primary aim, we tested whether we could identify immunogenic neoepitopes from ER+ BC with ER- BC and lung cancers as positive controls.

The Ensemble of Neopepsee and pVACseq predicted 93 neoepitopes from 299 somatic mutations in three ER+ BC patients. Among them, 90 could be tested with ELISpot, and 14 (15.6\%) were immunogenic (1, 5, and 10 for each tumor). In three ER- BC patients, 52 neoepitopes were predicted from 271 mutations, and $12(25.0 \%)$ of 48 tested were immunogenic $(2,4$, and 8 for each tumor). From three lung cancer PDXs, 53 from 72 predicted neoepitope candidates were tested, and 10 of them were immunogenic (18.9\%) (0, 1, and 11 for each tumor). These differences were not statistically significant. Therefore, we conclude that ER+ luminal B BCs express immunogenic neoepitopes, although their numbers vary widely between individual tumors. 
Table 3

Summary of ELISpot assays of IFN- $\mathbb{Z}$ secretion from neoantigen-specific T cell populations from donor PMBCs.

\begin{tabular}{|c|c|c|c|c|c|c|c|c|c|c|c|c|c|}
\hline & \multirow{2}{*}{$\begin{array}{l}\text { HLA } \\
\text { genotype }\end{array}$} & \multirow{2}{*}{$\begin{array}{l}\mathrm{N} \text { of } \\
\text { Mutations }\end{array}$} & \multicolumn{4}{|c|}{ NeoPepsee } & \multicolumn{4}{|c|}{ pVACseq (modified)* } & \multicolumn{3}{|c|}{ Common to both } \\
\hline & & & predicted & tested & ELISpotpositive & PPV & predicted & tested & $\begin{array}{l}\text { ELISPOT } \\
\text { positive }\end{array}$ & PPV & predicted & tested & $\begin{array}{l}\text { ELISpo } \\
\text { positive }\end{array}$ \\
\hline NE01 & $\begin{array}{l}A^{\star 2} 24: 02 \\
A^{\star} 33: 03\end{array}$ & 70 & 9 & 9 & 3 & 0.33 & 4 & 4 & 1 & 0.25 & 3 & 3 & 1 \\
\hline NEO2 &  & 94 & 7 & 6 & 2 & 0.33 & 7 & 6 & 0 & 0.00 & 1 & 0 & 0 \\
\hline NEO3 & $\begin{array}{l}A^{\star 02}: 06 \\
A^{\star} 11: 01\end{array}$ & 103 & 28 & 27 & 6 & 0.22 & 26 & 25 & 4 & 0.16 & 11 & 10 & 2 \\
\hline NEO4 & $\begin{array}{l}A^{*} 02: 06 \\
A^{*} 11: 01\end{array}$ & 96 & 33 & 31 & 1 & 0.03 & 3 & 3 & 0 & 0.00 & 1 & 1 & 0 \\
\hline NEO5 & $\begin{array}{l}A^{*} 02: 01 \\
A^{*} 02: 01\end{array}$ & 100 & 12 & 12 & 4 & 0.33 & 5 & 5 & 2 & 0.40 & 2 & 2 & 1 \\
\hline NEO6 & $\begin{array}{l}A^{\star} 02: 01 \\
A^{*} 24: 02\end{array}$ & 107 & 24 & 22 & 6 & 0.27 & 9 & 7 & 2 & 0.29 & 4 & 3 & 1 \\
\hline AV10 & $\begin{array}{l}A * 32: 01 \\
A * 33: 03\end{array}$ & 143 & 11 & 3 & 0 & 0.00 & 5 & 3 & 0 & 0.00 & 0 & 0 & 0 \\
\hline AV13 & $\begin{array}{l}A^{\star} 02: 01 \\
A^{\star} 30: 04^{\#}\end{array}$ & 109 & 21 & 17 & 6 & 0.35 & 15 & 13 & 5 & 0.38 & 4 & 2 & 2 \\
\hline AV38 & $\begin{array}{l}A^{*} 01: 01 \\
A^{*} 31: 01\end{array}$ & 76 & 14 & 10 & 0 & 0.00 & 10 & 9 & 1 & 0.11 & 0 & 0 & 0 \\
\hline Total & & 898 & 159 & 137 & 28 & 0.20 & 84 & 75 & 15 & 0.20 & 26 & 21 & 7 \\
\hline
\end{tabular}


Table 4

List of ELISpot-positive cancer-specific neoepitopes.

\begin{tabular}{|c|c|c|c|c|c|c|c|c|}
\hline case & $\begin{array}{l}\text { HLA } \\
\text { Allele }\end{array}$ & $\begin{array}{l}\text { Prediction } \\
\text { algorithm }\end{array}$ & $\begin{array}{l}\text { Candidate neoepitope ( } 9 \\
\text { or } 10 \mathrm{mer})\end{array}$ & $\begin{array}{l}\text { Wild type } \\
\text { sequence }\end{array}$ & $\begin{array}{l}\text { Gene } \\
\text { Symbol }\end{array}$ & $\begin{array}{l}\text { NetMHCpan } \\
\text { binding affinity } \\
\text { mutant }\end{array}$ & $\begin{array}{l}\text { NetMHCpan } \\
\text { binding affinity } \\
\text { Wild type }\end{array}$ & BindLevel \\
\hline Neo1 & $A * 24: 02$ & $\mathrm{~N}$ & SYGRLMFFC & SHGRLMFFC & CPA6 & 3276.94 & 24435.71 & \\
\hline $\mathrm{Neo1}$ & $A^{*} 24: 02$ & $\mathrm{~N}$ & RFIPGSSLL & RFIRGSSLL & ZNF517 & 126.35 & 173.14 & SB \\
\hline Neo1 & $A * 33: 03$ & NP & IYFLIGTSR & IYFLMGTSR & SLC26A1 & 40.73 & 55.21 & SB \\
\hline $\mathrm{Neo} 2$ & $A * 24: 02$ & $\mathrm{~N}$ & CYKMIGLTI & CYIMIGLTI & FAM162B & 162.07 & 142.00 & WB \\
\hline $\mathrm{Neo} 2$ & $A * 24: 02$ & $\mathrm{~N}$ & RYLQLQLHL & RYLQLQLYL & IKZF4 & 31.80 & 29.17 & SB \\
\hline $\mathrm{Neo3}$ & $A^{\star} 11: 01$ & $\mathrm{~N}$ & IAYNLYLIY & IAYNLSLIY & GTF3C3 & 195.64 & 115.19 & WB \\
\hline $\mathrm{Neo3}$ & $A^{\star} 11: 01$ & $\mathrm{~N}$ & ASVRKKLGK & ASVRKKLGE & SPTBN4 & 114.48 & 25706.04 & SB \\
\hline Neo3 & $A * 02: 06$ & $\mathrm{~N}$ & FLGSHLLHI & FLGSRLLHI & RTL1 & 11.48 & 33.03 & sB \\
\hline $\mathrm{Neo3}$ & $A * 11: 01$ & $\mathrm{~N}$ & PTTMPYPLK & PTTMTYPLK & VSIG4 & 495.86 & 589.41 & WB \\
\hline Neo3 & $A * 02: 06$ & NP & IILRALCAL & IILRAVCAL & SLFN5 & 56.88 & 134.81 & \\
\hline $\mathrm{Neo3}$ & $A^{\star} 11: 01$ & NP & (A)TACWSGLFK & (A)TACWSGLCK & PRPF4 & 22.26 & 166.48 & WB \\
\hline Neo3 & $A * 02: 06$ & $P$ & VLIKGSINSV & VLIEGSINSV & ARPC4 & 43.69 & 14.37 & SB \\
\hline Neo3 & $A * 11: 01$ & $P$ & HSNRLAVAYK & HTNRLAVAYK & DMXL1 & 18.12 & 12.66 & WB \\
\hline $\mathrm{Neo} 4$ & $A^{*} 02: 06$ & $\mathrm{~N}$ & YQDNVTIFA & YQDNVTVFA & ABCA2 & 30.64 & 31.39 & SB \\
\hline $\mathrm{Neo5}$ & $A * 02: 01$ & $\mathrm{~N}$ & LLAYSEYNL & LLAYSEYNLP & USP6 & 33.2 & 1872.93 & WB \\
\hline Neo5 & $A^{*} 02: 01$ & $\mathrm{~N}$ & AINSYRFLV & AINYYRFLV & GPR68 & 64.63 & 92.90 & WB \\
\hline Neo5 & $A * 02: 01$ & $\mathrm{~N}$ & KLQPFFEGM & KLKPFFEGM & PACS1 & 98.06 & 747.38 & SB \\
\hline Neo5 & $A^{*} 02: 01$ & NP & HLLQCAWLEI & HLLECAWLEI & AK9 & 99.66 & 18.76 & \\
\hline Neo5 & $A * 02: 01$ & $P$ & YMNAIKDYEL & YINAIKDYEL & PLEC & 8.21 & 106.30 & WB \\
\hline Neo6 & $A^{*} 24: 02$ & $\mathrm{~N}$ & YYQLFAATV & YYQLFAAAV & ANKRD53 & 45.53 & 285.69 & SB \\
\hline Neo6 & $A * 24: 02$ & $\mathrm{~N}$ & IYETNVVGF & IYETNVLGF & TULP1 & 157.53 & 92.89 & SB \\
\hline Neo6 & $A * 24: 02$ & $\mathrm{~N}$ & SFLKLAKLF & SFLKLAELF & SNX32 & 99.82 & 18.28 & SB \\
\hline Neo6 & $A^{\star 02: 01}$ & $\mathrm{~N}$ & YIQTTTLPV & YIQTTTLTV & GRM6 & 15.88 & 44.45 & WB \\
\hline Neo6 & $A^{\star} 24: 02$ & $\mathrm{~N}$ & YHIFFDQVF & YHIFFDKVF & PGBD2 & 812.59 & 2762.52 & WB \\
\hline Neo6 & $A \star 24: 02$ & NP & AYLPWSYFL & AYLPWSYFP & C4orf33 & 9.19 & 1014.65 & SB \\
\hline Neo6 & $A^{*} 02: 01$ & $P$ & KLFSRNSGL & KFFSRNSGL & ZNF304 & 55.09 & 9702.91 & SB \\
\hline AV13 & $A^{\star 02: 01}$ & $\mathrm{~N}$ & ALRRFAFMV & ALMRFAFMV & LPGAT1 & 171.47 & 3.52 & \\
\hline AV13 & $A^{*} 02: 01$ & $\mathrm{~N}$ & LLLFCDVGL & LLLFCDVDL & CHSY3 & 15.27 & 62.59 & WB \\
\hline AV13 & $A^{\star 02: 01}$ & $\mathrm{~N}$ & AMTILILKV & AMTIWILKV & SGCZ & 60.49 & 42.39 & SB \\
\hline AV13 & $A * 02: 01$ & $\mathrm{~N}$ & YLLMISALM & YLIMISALM & PSEN1 & 13.49 & 20.89 & WB \\
\hline AV13 & $A^{*} 02: 01$ & NP & LLLTCGEKV & LLLTCGEEV & STC2 & 54.01 & 24.09 & WB \\
\hline AV13 & $A^{\star 02: 01}$ & NP & GVANCLFPL & GVGNCLFPL & METTL6 & 21.62 & 91.66 & WB \\
\hline AV13 & $A^{\star} 02: 01$ & $P$ & FVIPEVFLKL & FVSPEVFLK & DNAAF5 & 64.82 & 10909.18 & WB \\
\hline AV13 & $A^{\star 02: 01}$ & $P$ & FLLRGPPVPV & FLLRGPPGPV & C8orf82 & 4.60 & 6.90 & SB \\
\hline AV13 & $A^{*} 02: 01$ & $P$ & YLQRNAPTL & YLQRNALTL & KDM6A & 21.75 & 35.01 & SB \\
\hline
\end{tabular}

Note: mutations and matching wild-type sequences are in bold character, $\mathrm{N}=$ predicted by NeoPepsee, $\mathrm{P}=$ predicted by $\mathrm{pVACseq}, \mathrm{NP}=\mathrm{predicted}$ by both NeoPepsee and pVACseq

For our first secondary aim of comparing the positive predictive value of Neopepsee versus pVACseq, 28 of 137 tested Neopepsee candidates were ELISpot positive (PPV 0.20) compared to 15 of 75 tested pVACseq candidates (PPV 0.20). These PPVs were not significantly different ( $p=0.95$ ). Among 21 tested candidates predicted by both algorithms, seven were positive by ELISpot (PPV 0.33). Therefore, we conclude that Neopepsee and pVACseq identify different pools of candidate neoepitopes from sequencing data and are complementary to each other, which in combination provide a reasonable number of candidates to screen for vaccine design, reducing the number of candidates that need to be screened experimentally from 8082 (898 times 9 possible positions for a mutation within a 9-mer nucleotide) to 217 with a median of 24 candidates per case (range 10 to 43 ) with a PPV of $18.8 \%$. 


\begin{tabular}{|c|c|c|c|c|c|c|c|c|}
\hline case & $\begin{array}{l}\text { HLA } \\
\text { Allele }\end{array}$ & $\begin{array}{l}\text { Prediction } \\
\text { algorithm }\end{array}$ & $\begin{array}{l}\text { Candidate neoepitope ( } 9 \\
\text { or } 10 \mathrm{mer})\end{array}$ & $\begin{array}{l}\text { Wild type } \\
\text { sequence }\end{array}$ & $\begin{array}{l}\text { Gene } \\
\text { Symbol }\end{array}$ & $\begin{array}{l}\text { NetMHCpan } \\
\text { binding affinity } \\
\text { mutant }\end{array}$ & $\begin{array}{l}\text { NetMHCpan } \\
\text { binding affinity } \\
\text { Wild type }\end{array}$ & BindLevel \\
\hline AV38 & $A * 31: 01$ & $\mathrm{P}$ & RQDIDFGVSR & RQDIDLGVSR & NFE2L2 & 49.25 & 115.92 & SB \\
\hline \multicolumn{9}{|c|}{$\begin{array}{l}\text { Note: mutations and matching wild-type sequences are in bold character, } \mathrm{N}=\text { predicted by NeoPepsee, } \mathrm{P}=\text { predicted by } \mathrm{pVACseq} \text {, NP=predicted by both } \\
\text { NeoPepsee and pVACseq }\end{array}$} \\
\hline \multicolumn{9}{|c|}{$\begin{array}{l}\text { For our first secondary aim of comparing the positive predictive value of Neopepsee versus pVACseq, } 28 \text { of } 137 \text { tested Neopepsee candidates were ELISpot } \\
\text { positive (PPV } 0.20 \text { ) compared to } 15 \text { of } 75 \text { tested pVACseq candidates (PPV } 0.20 \text { ). These PPVs were not significantly different ( } p=0.95 \text { ). Among } 21 \text { tested } \\
\text { candidates predicted by both algorithms, seven were positive by ELISpot (PPV } 0.33 \text { ). Therefore, we conclude that Neopepsee and pVACseq identify } \\
\text { different pools of candidate neoepitopes from sequencing data and are complementary to each other, which in combination provide a reasonable number } \\
\text { of candidates to screen for vaccine design, reducing the number of candidates that need to be screened experimentally from } 8082 \text { ( } 898 \text { times } 9 \text { possible } \\
\text { positions for a mutation within a 9-mer nucleotide) to } 217 \text { with a median of } 24 \text { candidates per case (range } 10 \text { to } 43 \text { ) with a PPV of } 18.8 \% \text {. }\end{array}$} \\
\hline
\end{tabular}

\section{Evaluation of TESLA recommended criteria for neoepitopes}

Previously, the Tumor Neoantigen Selection Alliance (TESLA) suggested that potential immunogenic peptides are characteristic of MHC binding affinity stronger than $34 \mathrm{nM}^{14}$. According to these criteria, we compared the validated neopeptides based on an MHC binding affinity of $34 \mathrm{nM}$ (Table 5 ).

Table 5

Summary of in vitro validated neopeptides by ELIspot assay according to MHC binding affinity of $34 \mathrm{nM}$

\begin{tabular}{|llll|}
\hline & IC $_{50}<34 \mathrm{nM}$ & $\mathrm{IC}_{50} \geq 34 \mathrm{nM}$ & Marginal row total \\
\hline ELISpot positive & $14(38.9 \%)$ & $22(61.1 \%)$ & 36 \\
\hline ELISpot negative & $47(30.3 \%)$ & $108(69.7 \%)$ & 155 \\
\hline Marginal column total & $61(31.9 \%)$ & $130(68.1 \%)$ & 191 \\
\hline
\end{tabular}

Fourteen of $36(38.9 \%)$ ELISpot-positive peptides had NetMHCpan IC $_{50}$ below 34 nM, whereas 47 of 155 (30.3\%) ELISpot-negative peptides had IC 50 below 34 $\mathrm{nm}$ (Fisher's exact test, $p=0.32$, NS). If we were to predict based on the $\mathrm{IC}_{50}$ value, $61 / 191(31.9 \%)$ peptides were predicted to be high-affinity binders, and among them, 14 (22.9\%) were true positives. Therefore, we could not corroborate the findings from the TESLA consortium.

\section{Discussion}

Clinical trials have demonstrated that therapeutic vaccines targeting neoepitopes derived from somatic mutations are promising therapeutic modalities for solid tumors with a high mutation burden. ${ }^{8-11}$ However, with a small sample size, we demonstrated that luminal B breast cancer does express at least one or more immunogenic neoepitopes. Since there is little therapeutic option for luminal B BC patients after distant recurrence, it is worth considering them for inclusion in therapeutic vaccine trials. Epitope spreading after therapeutic neoepitope vaccination suggests that immune ignorance may be one of the immune evasion mechanisms. ${ }^{35} \mathrm{~A}$ study by Westcott et al suggested that for patients with immune ignorance of clonal neoepitopes due to low level expression, therapeutic vaccination of neoepitopes may be able to trigger robust immune responses. ${ }^{36}$

Clinical trials for personalized therapeutic neoepitope vaccines have demonstrated that T-cell immune responses were induced for only a subset of candidate neoepitopes. ${ }^{8-11}$ Although almost all vaccine trials reported thus far selected candidates based on computational algorithms, one trial selected candidates based on in vitro T-cell response to candidate neoepitopes presented by bacteria. ${ }^{37}$ Intriguingly, in the latter study, some mutated peptides suppressed the immune response, and investigators named such peptides 'inhibigens'. Inhibigens suppressed immune activation by otherwise immune-stimulatory neoepitopes when injected together. Since in vitro validation of every candidate neoepitope is demanding and time-consuming, it is challenging to incorporate it into a routine process. Thus, designing personalized therapeutic vaccines requires a robust computational neoepitope prediction algorithm with a high positive predictive value, ideally followed by in vitro testing of immunogenic responses with patient or HLA-matched donor PBMCs.

In 9 cases prospectively screened using the ELISpot assay with HLA-matched donor PBMCs, the PPV was $20 \%$ for both Neopepsee and pVACseq. There was relatively little overlap between the predicted epitopes between the two algorithms. The PPV increased to $33 \%$ for overlapping candidates between Neopepsee and pVACseq. This finding is consistent with the TESLA report in that ensemble models outperform individual prediction models. The ensemble of Neopepsee and pVACseq provided a reasonable number of candidates to screen for vaccine design, reducing the number of candidates that need to be screened experimentally from 8082 (898 times 9 possible positions for a mutation within a 9-mer nucleotide) to 217 with a median of 24 candidates per case (range 10 to 43 ) with a PPV of $18.8 \%$. However, it would be ideal to validate the immunogenicity of the predicted neoepitope candidates with an in vitro screening method before designing a therapeutic vaccine.

TESLA identified a set of thresholds for several variables that in combination could filter out $93 \%$ of nonimmunogenic peptides while maintaining $55 \%$ of immunogenic peptides. ${ }^{24}$ This threshold set is composed of binding affinity less than $34 \mathrm{nM}$, tumor abundance greater than 33 TPM, and binding stability greater than $1.4 \mathrm{~h}$. We could not corroborate the findings from the TESLA consortium. Unlike TESLA, which used a multimer assay to validate the neoepitopes, we used an ELISpot assay. While a multimer assay quantifies T-cells with T-cell receptors that can bind MHC-presented neoepitopes, the ELISpot assay quantifies the interferon- $\varangle$ secreted by the T-cells activated by the MHC-presented neoepitopes. The differences in sensitivity and specificity between the two assays may be partially responsible for the observed difference with the TESLA data. 
Our study has several limitations: 1) We did not screen mutated peptides that were not predicted by Neopepsee or pVACseq. Since there is a possibility that some of them might be immunogenic, we do not know the true false-negative rate of each algorithm. 2) We used HLA-matched donor blood for the ELISpot assay. Stronen et al. reported that HLA-matched healthy donor blood showed a five times higher response rate than blood from patients. ${ }^{25}$ Thus, our result of $20 \%$ PPV could be an overestimate. 3) For the ELISpot assay, we used strict criteria of requiring a memory response upon rechallenge with the candidate peptides after ten days of incubation with the peptides. Some of the peptides showed increased spot numbers over control wells that had never contacted the peptides but did not show increased spot numbers in comparison to the no peptide control at the time of rechallenge. Such results could be interpreted as positive responses by some investigators. 4) ELISpot is known to be influenced by many factors. ${ }^{38}$ Compared to viral antigens, which show strong positive ELISpot results, most of the neoepitopes we found showed only a moderate increase in spot numbers. Since we did not validate our results with a multimer assay, some peptides could be false-positive findings.

In conclusion, our data demonstrate that luminal B BC can be immunogenic and replicate the findings from many studies that the current generation of neoepitope prediction algorithms suffers from low PPV, and more in vitro validation data are required to train the prediction algorithms to achieve clinically meaningful PPV. In the absence of prediction algorithms with high PPV, it may make sense to design vaccines based on in vitro validated peptides only. For the latter aim, developing robust and cost-efficient methods for real-time in vitro screening of large numbers of candidate neoepitopes is necessary. For example, although a tandem minigene screen allows screening of 10 mutated genes in a single construct and thus allows experimental screening of all mutations with ELISpot, individual peptides eventually need to be synthesized to pinpoint the neoepitope within the positive minigene. While several innovative high-throughput screening methods have been reported recently, they do not fulfill such needs due to the high cost of peptide synthesis required for the assay or due to low sensitivity. ${ }^{39-41}$

\section{References}

1. Hanahan, D. \& Weinberg, R. A. Hallmarks of Cancer: The Next Generation. Cell 144, 646-674 (2011).

2. Vogelstein, B. et al. Cancer Genome Landscapes. Science 339, 1546-1558 (2013).

3. Tran, E., Robbins, P. F. \& Rosenberg, S. A. "Final common pathway" of human cancer immunotherapy: targeting random somatic mutations. Nat Immunol 18, 255-262 (2017).

4. Blass, E. \& Ott, P. A. Advances in the development of personalized neoantigen-based therapeutic cancer vaccines. Nat Rev Clin Onco/ 18, 215-229 (2021).

5. Gupta, R. G., Li, F., Roszik, J. \& Lizée, G. Exploiting Tumor Neoantigens to Target Cancer Evolution: Current Challenges and Promising Therapeutic Approaches. Cancer Discov 11, 1024-1039 (2021).

6. Jou, J., Harrington, K. J., Zocca, M.-B., Ehrnrooth, E. \& Cohen, E. E. W. The Changing Landscape of Therapeutic Cancer Vaccines-Novel Platforms and Neoantigen Identification. Clin Cancer Res 27, 689-703 (2021).

7. Zacharakis, N. et al. Immune recognition of somatic mutations leading to complete durable regression in metastatic breast cancer. Nat Med $\mathbf{2 4}$, 724-730 (2018).

8. Linette, G. P. \& Carreno, B. M. Neoantigen Vaccines Pass the Immunogenicity Test. Trends Mol Med 23, 869-871 (2017).

9. Carreno, B. M. et al. A dendritic cell vaccine increases the breadth and diversity of melanoma neoantigen-specific T cells. Science 348, 803-808 (2015).

10. Ott, P. A. et al. An immunogenic personal neoantigen vaccine for patients with melanoma. Nature 547, 217-221 (2017).

11. Sahin, U. et al. Personalized RNA mutanome vaccines mobilize poly-specific therapeutic immunity against cancer. Nature 547, 222-226 (2017).

12. Supabphol, S., Li, L., Goedegebuure, S. P. \& Gillanders, W. E. Neoantigen vaccine platforms in clinical development: understanding the future of

personalized immunotherapy. Expert Opin Inv Drug 00, 1-13 (2021).

13. Thorsson, V. et al. The Immune Landscape of Cancer. Immunity 48, 812-830.e14 (2018).

14. Teku, G. N. \& Vihinen, M. Pan-cancer analysis of neoepitopes. Sci Rep-uk 8, 12735 (2018).

15. Turajlic, S. et al. Insertion-and-deletion-derived tumour-specific neoantigens and the immunogenic phenotype: a pan-cancer analysis. Lancet Onco/ 18, 1009-1021 (2017).

16. Perou, C. M. et al. Molecular portraits of human breast tumours. Nature 406, 747-752 (2000).

17. Paik, S. et al. A Multigene Assay to Predict Recurrence of Tamoxifen-Treated, Node-Negative Breast Cancer. New Engl J Medicine 351, $2817-2826$ (2004).

18. Paik, S. et al. Gene Expression and Benefit of Chemotherapy in Women With Node-Negative, Estrogen Receptor-Positive Breast Cancer. J Clin Oncol 24, 3726-3734 (2006).

19. Sparano, J. A. et al. Adjuvant Chemotherapy Guided by a 21-Gene Expression Assay in Breast Cancer. New Engl J Med 379, 111-121 (2018).

20. Sopik, V., Sun, P. \& Narod, S. A. Predictors of time to death after distant recurrence in breast cancer patients. Breast Cancer Res Tr 173, 465-474 (2019).

21. Reimann, $H$. et al. Identification and validation of expressed HLA-binding breast cancer neoepitopes for potential use in individualized cancer therapy. $J$ Immunother Cancer 9, e002605 (2021).

22. Morisaki, T. et al. Neoantigens elicit T cell responses in breast cancer. Sci Rep-uk 11, 13590 (2021).

23. Narang, P., Chen, M., Sharma, A. A., Anderson, K. S. \& Wilson, M. A. The neoepitope landscape of breast cancer: implications for immunotherapy. Bmc Cancer 19, 200 (2019).

24. Wells, D. K. et al. Key Parameters of Tumor Epitope Immunogenicity Revealed Through a Consortium Approach Improve Neoantigen Prediction. Cell 183, 818-834.e13 (2020).

25. Strønen, E. et al. Targeting of cancer neoantigens with donor-derived T cell receptor repertoires. Science 352, 1337-1341 (2016).

26. Czerkinsky, C. C., Nilsson, L.-Å., Nygren, H., Ouchterlony, Ö. \& Tarkowski, A. A solid-phase enzyme-linked immunospot (ELISPOT) assay for enumeration of specific antibody-secreting cells. J Immunol Methods 65, 109-121 (1983).

27. Kim, S. et al. Neopepsee: accurate genome-level prediction of neoantigens by harnessing sequence and amino acid immunogenicity information. Ann

Oncol 29, 1030-1036 (2018). 
28. Hundal, J. et al. pVAC-Seq: A genome-guided in silico approach to identifying tumor neoantigens. Genome Med 8, 11 (2016).

29. Szolek, A. et al. OptiType: precision HLA typing from next-generation sequencing data. Bioinformatics 30, 3310-3316 (2014).

30. Warren, R. L. et al. Derivation of HLA types from shotgun sequence datasets. Genome Med 4, 95-95 (2012).

31. Li, H. Aligning sequence reads, clone sequences and assembly contigs with BWA-MEM. Arxiv (2013).

32. Jo, S.-Y., Kim, E. \& Kim, S. Impact of mouse contamination in genomic profiling of patient-derived models and best practice for robust analysis. Genome Biol 20, 231 (2019).

33. Auwera, G. A. V. der \& O'Connor, B. D. Genomics in the Cloud: Using Docker, GATK, and WDL in Terra (1st Edition). (O'Reilly Media, Inc., 2020).

34. Karczewski, K. J. et al. The mutational constraint spectrum quantified from variation in 141,456 humans. Nature 581, 434-443 (2020).

35. Linette, G. P. et al. Immunological ignorance is an enabling feature of the oligo-clonal T cell response to melanoma neoantigens. Proc National Acad Sci 116, 23662-23670 (2019).

36. Westcott, P. M. K. et al. Low neoantigen expression and poor T-cell priming underlie early immune escape in colorectal cancer. Nat Cancer 1-15 (2021) doi:10.1038/s43018-021-00247-z.

37. Lam, H. et al. An Empirical Antigen Selection Method Identifies Neoantigens That Either Elicit Broad Antitumor T-cell Responses or Drive Tumor Growth. Cancer Discov 11, 696-713 (2021).

38. Janetzki, S. et al. Results and harmonization guidelines from two large-scale international Elispot proficiency panels conducted by the Cancer Vaccine Consortium (CVC/SVI). Cancer Immunol Immunother 57, 303-315 (2008).

39. Saini, S. K. et al. Empty peptide-receptive MHC class I molecules for efficient detection of antigen-specific T cells. Sci Immuno/ 4, eaau9039 (2019).

40. Kula, T. et al. T-Scan: A Genome-wide Method for the Systematic Discovery of T Cell Epitopes. Cell 178, 1016-1028.e13 (2019).

41. Lee, M. N. \& Meyerson, M. Antigen identification for HLA class I- and HLA class II-restricted T cell receptors using cytokine-capturing antigen-presenting cells. Sci Immunol 6, eabf4001 (2021).

\section{Supplementary Files}

This is a list of supplementary files associated with this preprint. Click to download.

- supplementarytable1.xIsx 